\section{IN THE NEWS}

Out-of-body experience "New out-of-body operation saves cancer patient" was the headline in The Times, UK (19 December 2002). At the San Matteo Hospital in Pavia, Italy, surgeons treated a man who had metastatic cancer in his liver by removing the organ, treating it with radiotherapy, and then re-implanting it. The operation carried out late in 2001 took 21 hours.

One year on, the patient is alive and well with no signs of tumour recurrence, so the surgeons are now planning to treat six more patients who have multiple liver tumours that are resistant to chemotherapy and would not benefit from conventional radiotherapy, reported the New Scientist (18 December 2002).

The team used boron neutron capture therapy - a technique developed in the 1950s that has proved difficult to use because the neutron beam is easily blocked from reaching the tumour by obstructions such as bones, and because of toxicity to normal tissues. "By explanting the organ, we could give a high and uniform dose to all the liver, which is impossible to obtain inside the body without serious risk to the patient", says Tazio Pinelli, a physicist who worked with the liver surgeon Aris Zonta. This technique could one day be used to treat tumours in other organs that can be transplanted, such as the pancreas or lungs. Boron neutron capture therapy is also currently in Phase I trials for untreatable brain tumours - "obviously without removing the organ in question", says the New Scientist

\title{
TELOMERE MAINTENANCE
}

\section{The road less travelled}

As aspiring cancer cells progress along the road to malignancy, they are presented with many choices, among which are the mechanisms used to maintain telomeres and hence chromosomal integrity reactivation of telomerase or the recombinationmediated ALT mechanism. The prevailing view has held that these alternative mechanisms represent equally viable options; however, recent genetic studies have pointed to important biological differences with therapeutic implications. A study in the 1 January issue of Genes \& Development by Ron DePinho and colleagues reveals that telomerase, but not ALT, enables would-be cancer cells to achieve a full malignant end point, which includes metastasis.

DePinho and colleagues generated two sets of mouse embryonic fibroblasts (MEFs) to investigate the contribution of telomere-based crisis and telomerase to tumour progression. Fifth-generation $\mathrm{Terc}^{--} \mathrm{Cdkn} 2 \mathrm{a}^{-1-}$ mice were transfected with $M Y C$ and $R A S$, and then with either an empty vector $(\mathrm{V})$ or $\operatorname{Terc}(\mathrm{T})$, which restored telomerase activity. Although the two lines initially behaved similarly in culture, prolonged passage resulted in a decreased growth rate in only the $\mathrm{V}$ cell line. Addition of Terc to late-passage $\mathrm{V}$ lines was able to increase the growth rate, indicating that the presence of telomerase was responsible for this difference.

Cytogenetic analysis also revealed that $\mathrm{T}$ lines had a low number of chromosomal fusions and other aberrations, whereas $\mathrm{V}$ lines showed a steady increase in abnormalities as they advanced through crisis and experienced further telomere erosion.

So how do these chromosomal abnormalities affect the ability to form tumours? Telomerase enhances growth and promotes immortality, but chromosomal abnormalities could generate genomic instability, which facilitates the initial stages of tumour development. Early-, mid- and late-passage MEFs from the $V$ and $T$ lines were injected subcutaneously and into the tail vein, to investigate tumour growth and metastatic potential, respectively. The $T$ lines grew well subcutaneously, and metastatic lung tumour nodules - which were high grade and occupied $20-80 \%$ of lung volume - had formed by 5 weeks post-injection. The $\mathrm{V}$ lines behaved differently; although they were able to grow subcutaneously albeit at a slower rate than the $\mathrm{T}$ lines - metastatic potential was severely compromised in the $\mathrm{V}$ cells. Early-passage $\mathrm{V}$ cells produced small lesions in the lungs, but they were less aggressive - mice stayed healthy for longer. Mid- and late-passage cells also seeded to the lungs, but were unable to grow beyond microscopic lesions. To investigate whether lack of telomerase was directly responsible for this difference in potential, telomerase activity was reconstituted by transfecting with Terc - in early-, mid- and latepassage $\mathrm{V}$ cells. This restored the metastatic potential,

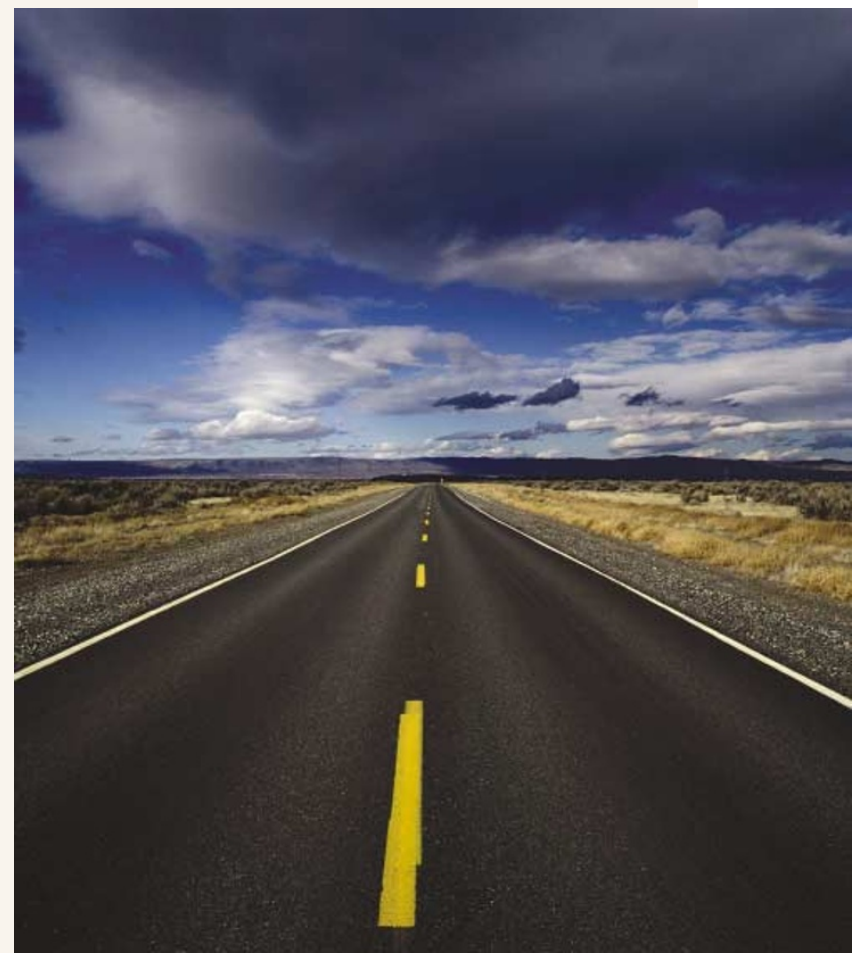

indicating that telomerase can directly promote tumour progression.

As the $\mathrm{V}$ lines are able to sustain growth with shortened telomeres, might they be able to activate ALT? The sarcomas that were formed from the $\mathrm{V}$ cells were used to generate more tumours in immunodeficient mice. These were more aggressive and had a significantly faster doubling time -12 hours compared with 29 hours. The telomeres were long and heterogeneous - which is a characteristic of ALT and its presence was confirmed when ALT-associated PML bodies were detected. So, extensively passaged $\mathrm{V}$ lines can activate ALT under the biological pressure of tumorigenesis, and this promotes growth and tumour potential. However, unexpectedly, ALT fails to restore metastatic potential.

So, these results reveal that functional differences exist between ALT and telomerase, and directly implicate telomerase in enhancing metastatic potential. They also quell concerns that ALT-mediated telomere maintenance might provide cancer cells with a robust resistance mechanism against anti-telomerase cancer therapy.

Emma Greenwood

6) References and links

ORIGINAL RESEARCH PAPER Chang. S. et al. Telomere-based crisis: functional differences between telomerase activation and ALT in tumor progression. Genes Dev. 17, 88-100 (2003) WEB SITE

Ron DePinho's lab:

http://www.hms.harvard.edu/dms/bbs/fac/depinho.html 